Gynecology

\title{
Examining trends in non-fatal strangulation among sexual assault survivors seeking Sexual Assault Nurse Examiner care from 2002 to 2017
}

\author{
Lindsay M. Cannon ${ }^{1,2, *}$ | Joanne M. Bailey ${ }^{3}$ | Susan D. Ernst ${ }^{4}$ | Amanda St. Ivany ${ }^{5}$ | \\ Kristin G. Bevilacqua ${ }^{6}$ | Lisa Scheiman ${ }^{3}$ | Michelle L. Munro-Kramer ${ }^{7}$
}

\author{
${ }^{1}$ Department of Sociology, University of Wisconsin - Madison, Madison, WI, USA \\ ${ }^{2}$ Center for Demography and Ecology, University of Wisconsin - Madison, Madison, WI, USA \\ ${ }^{3}$ Michigan Medicine Nurse-Midwifery Service, Department of Obstetrics and Gynecology, University of Michigan, Ann Arbor, MI, USA \\ ${ }^{4}$ University Health Service, University of Michigan, Ann Arbor, MI, USA \\ ${ }^{5}$ Department of Community and Family Medicine, Geisel School of Medicine at Dartmouth College, Lebanon, NH, USA \\ ${ }^{6}$ Department of Population, Family and Reproductive Health, Johns Hopkins School of Public Health, Baltimore, MD, USA \\ ${ }^{7}$ School of Nursing, University of Michigan, Ann Arbor, MI, USA \\ ${ }^{*}$ Correspondence \\ Lindsay M. Cannon, Department of Sociology, Center for Demography and Ecology, University of Wisconsin - Madison, Madison, WI, USA. \\ Email: Imcannon@wisc.edu
}

KEYWORDS: Non-fatal strangulation; Sexual assault; Sexual assault nurse examiner; United States

Non-fatal strangulation (NFS) is a global public health and human rights issue. ${ }^{1}$ Prevalence estimates of NFS in sexual assault (SA) range from $7.4 \%$ to $12 \%{ }^{2,3}$ Strangulation associated with SA poses a lethality risk, both at the time of assault and through risk for future strangulation. If the assailant is an intimate partner, a heightened risk exists for future attempted and completed homicide. ${ }^{4}$

This study explores prevalence, trends, and characteristics of SA involving NFS using a retrospective descriptive analysis of Sexual Assault Nurse Examiner (SANE) cases at one academic hospital in the Midwestern United States from 2002 to 2017. Data were extracted from systematic statewide SANE data collection forms and SANE notes for females after menarche and males $\geq 13$ years (considered adults through the hospital protocol). The institutional review board of the hospital approved the study procedures. Descriptive statistics and chi-square tests were computed in SPSS version 25 (IBM, Armonk, NY, USA); $P<0.05$ were considered significant.

Of 856 cases, $95.7 \%$ of survivors were female and $61.1 \%$ were white. The average age was 24.16 years. In total, $5.1 \%$ of cases involved NFS. All NFS survivors were female, with $11.3 \%$ of females experiencing NFS. NFS was more common when current/former partners $(18.9 \%)$ or strangers were assailants $(16.6 \%)$, compared to family members $(11.1 \%)$ or friends/acquaintances $\left(7.9 \% ; \chi^{2}(4)=9.72\right.$, $P=0.045)$. NFS occurred more often in cases involving weapons than in cases without weapons $\left(30.0 \%\right.$ vs. $\left.9.1 \% ; \chi^{2}(1)=15.50, P<0.001\right)$. Additionally, NFS occurred more frequently in cases with anal penetration than cases without $\left(22.7 \%\right.$ versus $\left.7.5 \% ; \chi^{2}(1)=16.19, P<0.001\right)$. NFS appears to increase over time (Fig. 1). The mean number of NFS cases annually was 1.8 from 2002 to 2007 (range 0-3), 2.4 from 2008 to 2012 (range 1-5), and 4.6 from 2013 to 2017 (range 3-6).

These results indicate that NFS is common in SA involving females. Our findings are consistent with research showing that survivors experiencing SA by current/former partners are at an increased risk of lethality, ${ }^{2,3}$ followed by SA perpetrated by strangers. Furthermore, many survivors face a heightened risk of lethality through the combination of weapons and strangulation. It is possible that NFS is increasing, but more data are needed. This study was limited by the

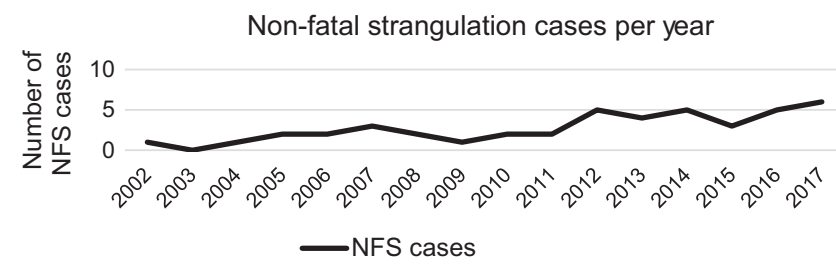

FIGURE 1 Number of non-fatal strangulation cases associated with sexual assault per year (2002-2017). 
small number of NFS cases and data from one hospital, which may not be representative of cases at other health centers. However, the strengths include the large sample size and systematic data collection. These results highlight the importance of screening for strangulation when survivors of SA present for care and the need to tailor interventions based on lethality risk.

\section{AUTHOR CONTRIBUTIONS}

LMC conceived the study, compiled and analyzed the data, and wrote the first draft of the manuscript. JMB, SDE, ASI, \& LS conceived the study and revised the manuscript. KGB compiled the data and revised the manuscript. MLMK conceived the study, compiled and analyzed the data, and revised the manuscript.

\section{CONFLICTS OF INTEREST}

The authors have no conflicts of interest to disclose.

\section{REFERENCES}

1. Sorenson SB, Joshi M, Sivitz E. A systematic review of the epidemiology of nonfatal strangulation, a human rights and health concern. Am J Public Health. 2014;104:e54-e61.

2. Zilkens RR, Phillips MA, Kelly MC, Mukhtar SA, Semmens JB, Smith DA. Non-fatal strangulation in sexual assault: A study of clinical and assault characteristics highlighting the role of intimate partner violence. J Forensic Leg Med. 2016;43:1-7.

3. Mcquown C, Frey J, Steer S, et al. Prevalence of strangulation in sexual assault and domestic violence. Acad Emerg Med. 2016;34:1281-1285.

4. Glass N, Laughon K, Campbell J, et al. Non-fatal strangulation is an important risk factor for homicide of women. J Emerg Med. 2008;35:329-335.

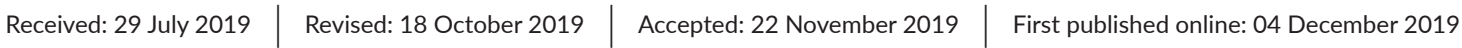

DOI: $10.1002 /$ ijgo.13066

Gynecology

\title{
Pelvic inflammatory disease and risk of cancer: A nationwide cohort study
}

\author{
Kirstine K. Søgaard ${ }^{1, *}$ | Michelle Z. Leisner ${ }^{1}$ | Kristina Laugesen ${ }^{1}$ | \\ Dóra K. Farkas $^{1}$ | Margaret R. Karagas ${ }^{2}$ | Henrik T. Sørensen ${ }^{1}$
}

\author{
${ }^{1}$ Department of Clinical Epidemiology, Aarhus University Hospital, Aarhus, Denmark \\ ${ }^{2}$ Department of Epidemiology, Geisel School of Medicine, Dartmouth College, Lebanon, NH, USA \\ ${ }^{*}$ Correspondence \\ Kirstine Kobberøe Søgaard, Department of Clinical Epidemiology, Aarhus University Hospital, Aarhus, Denmark. \\ Email: kks@clin.au.dk
}

KEYWORDS: Cancer; Epidemiology; Incidence; Pelvic inflammatory disease

Pelvic inflammatory disease (PID) is associated with an increased risk of subsequent reproductive sequelae and possibly an increased incidence of endometrial, serous ovarian, and colorectal cancer. ${ }^{1-3}$ Any association with cancer may have important public health implications. The present study examined the incidence of cancer among women with PID.

In this Danish population-based cohort study, we linked data from the National Patient Registry, ${ }^{4}$ the Civil Registration System, and the Cancer Registry. We identified females ( $\geq 15$ years) with incident PID diagnosed during 1978-2013 (using International Classification of Diseases, 8th and 10th Revision), ${ }^{4}$ and followed them for subsequent records of cancer diagnosis, death, emigration, or until termination of study (November 30, 2013). Women previously diagnosed with cancer were excluded from the present study.
We computed standardized incidence ratios (SIRs) and 95\% confidence intervals ( $\mathrm{Cls}$ ) to compare the observed against the expected number of cancers, estimating SIRs $<1$ year and $\geq 1$ year from PID diagnosis. Expected numbers were calculated on the basis of national cancer incidence rates, using age and calendar period (5-year intervals). According to Danish law, use of registry data for research purposes does not require informed consent.

Among 93706 females with PID, 542 cancers were diagnosed $<1$ year after a PID diagnosis, and 8727 cancers $>1$ year following a PID diagnosis. The SIR of any cancer $<1$ year following a PID diagnosis was 2.9 (95\% Cl; 2.7-3.2), with markedly elevated SIRs for urogenital and colorectal cancer. While most cancers were diagnosed among women over the age of 35, the SIR of any cancer was elevated for all 
TABLE 1 Standardized incidence ratios ${ }^{\mathrm{a}}$ for selected cancers ${ }^{\mathrm{b}}$ among 93706 women with pelvic inflammatory disease (Denmark, 1978-2013).

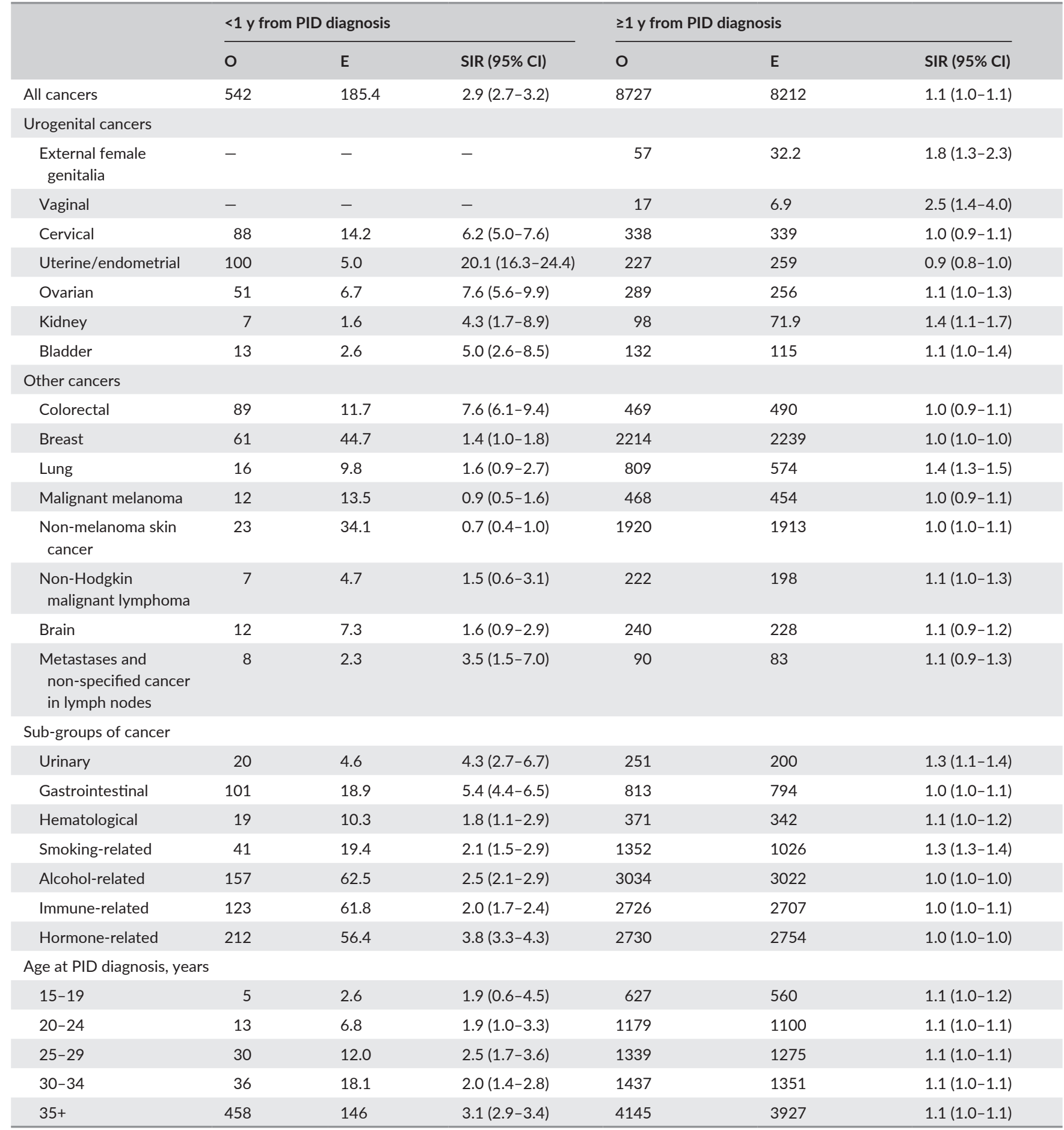

Abbreviations: E, expected; O, observed; PID, pelvic inflammatory disease; SIR, standardized incidence ratio.

The age distribution of the cohort: $15-19$ years: $n=13394$ (14\%); 20-24 years: $n=19514$ (21\%); 25-29 years: $n=17245$ (18\%); $30-34$ years: $n=14$ 697 (16\%); $35+$ years: $n=28856$ (31\%).

andirect standardized incidence ratios (SIRs) were used as a measure of relative risk by comparing the cancer incidence observed among women with PID with the expected incidence in the general Danish female population. The observed number of a specific cancer subtype was compared with the expected number of that specific subtype.

${ }^{b}$ In agreement with Danish data security regulations, the study only presents results for cancer sites with five or more cases. 
age groups $>20$ years. Beyond 1 year of follow-up, there was no association with cancer in general; however, individual cancers including kidney, lung, vaginal, and external female genitalia cancer remained higher than expected (Table 1).

The present study found a higher occurrence of several cancers within the first year after PID diagnosis, possibly explained by prevalent cancers detected during work-up for PID. The data suggested that some urogenital cancers may be overlooked at the time of PID diagnosis.

The strengths of the present study include the large sample size and virtually complete follow-up. The population-based registries are complete and have high data validity. ${ }^{4}$ Nevertheless, non-differential misclassification of patients could have resulted in biases toward the null. Moreover, individuals with subclinical PID, or a severity not requiring hospitalization, were not included in our cohort. Thus, the results may only apply to patients diagnosed with PID during hospital contact.

We disaggregated follow-up into $<1$ and $\geq 1$ year to discern prevalent versus incident cases of cancer. Therefore, the findings of the present study do not suggest causality between PID and cancer. However, undetected urogenital and colorectal cancer may mimic or cause PID, and may be considered as a differential diagnosis of PID in women older than 35 years diagnosed during hospital contact.

\section{AUTHOR CONTRIBUTIONS}

KKS, MZL, DKF, and HTS designed the study; DKF and HTS obtained and assembled the data; KKS, MZL, KL, DKF, MRK and HTS analyzed and interpreted the data; KKS and MZL wrote the report; and all authors revised the report and approved the final version of the manuscript.

\section{CONFLICTS OF INTEREST}

The authors have no conflicts of interest.

\section{REFERENCES}

1. Yang TK, Chung CJ, Chung SD, Muo $\mathrm{CH}$, Chang $\mathrm{CH}$, Huang CY. Risk of endometrial cancer in women with pelvic inflammatory disease: $\mathrm{A}$ nationwide population-based retrospective cohort study. Medicine (Baltimore). 2015;94:e1278.

2. Hsu MI, Lin HW. Risk of colorectal cancer in women with pelvic inflammatory disease: A matched cohort study. BJOG. 2014;121:337-342.

3. Rasmussen CB, Jensen A, Albieri V, Andersen KK, Kjaer SK. Is pelvic inflammatory disease a risk factor for ovarian cancer? Cancer Epidemiol Biomarkers Prev. 2017;26:104-109.

4. Schmidt M, Schmidt SA, Sandegaard JL, Ehrenstein V, Pedersen L, Sørensen HT. The Danish National Patient Registry: A review of content, data quality, and research potential. Clin Epidemiol. 2015;7:449-490.

\section{A global strategy to increase uptake and acceptability of LARC in a low-resource setting}

\section{Zainab Ahmed* | Taraneh Shirazian}

Saving Mothers, New York, NY, USA

*Correspondence

Zainab Ahmed, Saving Mothers, New York, NY, USA.

Email: zainab.ahmed@icahn.mssm.edu

KEYWORDS: Acceptability; Care model; Family planning; Kenya; LARC; Maternal mortality

In Kenya, rural communities bear the greatest burden of maternal deaths. In West Pokot, there is an average of 7.2 children per mother compared to 2.7 in Nairobi, with a contraception prevalence of $14 \%$ versus $63 \%$ in the capital. ${ }^{1}$ Access, cost and cultural beliefs are barriers to long-acting reversible contraceptive (LARC) uptake. ${ }^{2}$ The state of women's health in West Pokot presents a unique opportunity to reduce maternal mortality through family planning. The purpose of this ongoing prospective cohort study is to encourage childbirth spacing and develop trust in the uptake of LARCs in a low-resource, high-risk setting. IRB ethical approval was obtained in 2015 from the 


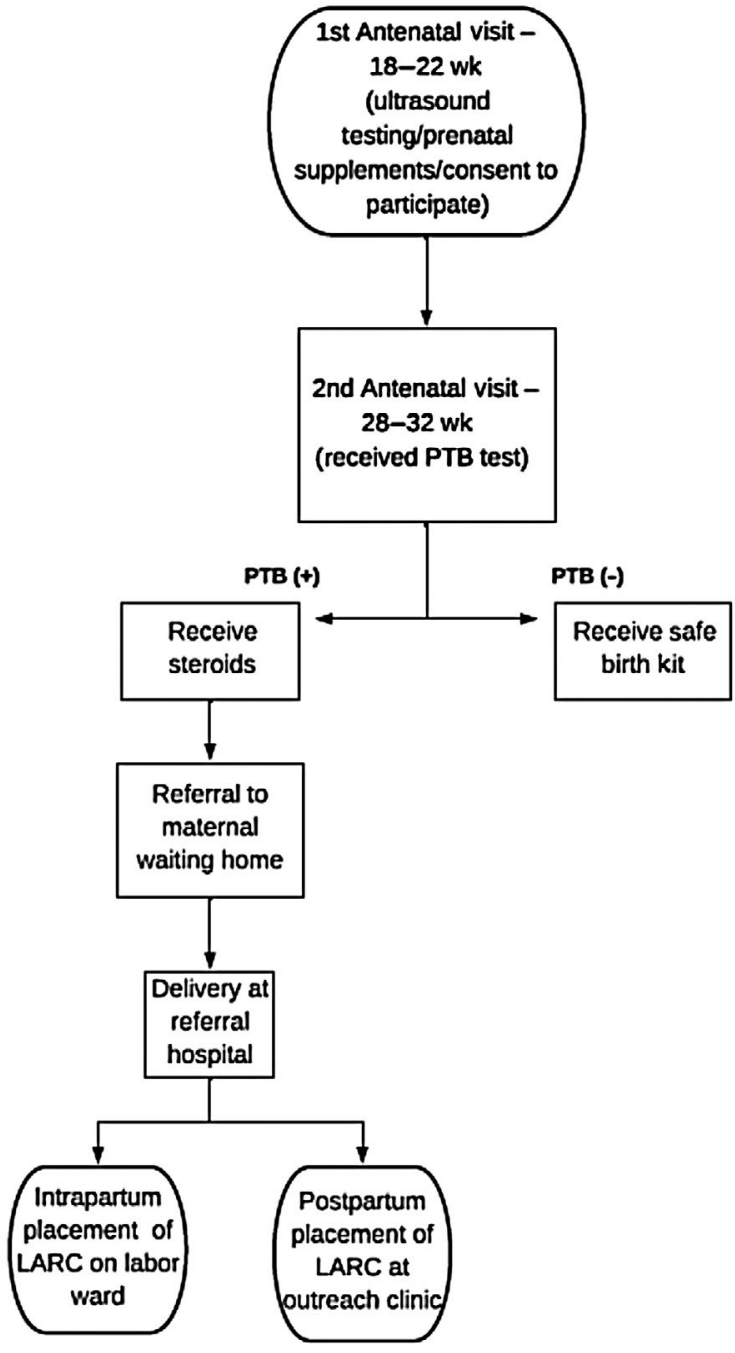

FIGURE 1 Saving Mothers' Maternal Continuum of Care Program.

Kenyan government through the Kenyatta National Hospital and the University of Nairobi.

All obstetric patients entering Kapenguria Hospital or other satellite clinics for prenatal care prior to 22 weeks of gestation were offered enrollment in the maternal continuum of care program (MCCP) (Fig. 1). The MCCP consists of three distinct prenatal visits. Consent was coordinated in Pokot or Swahilli by a local program coordinator and patients officially enrolled by signature or thumbprint at their first prenatal visit with ultrasound scan. After a second visit for preterm birth indicators, free LARCs were offered and the option of postpartum placement was discussed. Surveys about LARCs were administered prior to insertion, either following the delivery at the hospital or postpartum. Acceptability surveys were administered more than 14 months post-placement. $^{3}$

A total of 260 pregnant women consented to enrollment in the MCCP. All patients were offered contraceptive counseling and 139 accepted LARC placement; 59 (42\%) opted for levonorgestrel IUD and 55 (40\%) chose the etonogestrel implant. Mean age was 29.8 years and mean parity was 3 (range: 0-10). Seventy-nine (57\%) cited localized hormonal effects and 26 (19\%) reported
TABLE 1 Acceptability survey results.

\begin{tabular}{|lcc|}
\hline & $\mathrm{N}=\mathbf{3 0}$ & $\%$ \\
\hline Age & & \\
\hline$<25$ & 3 & $10 \%$ \\
\hline $26-30$ & 8 & $27 \%$ \\
\hline $31-35$ & 6 & $20 \%$ \\
\hline$>35$ & 13 & $43 \%$ \\
\hline Method chosen & & \\
\hline Copper IUD & 16 & $53 \%$ \\
\hline LNG IUD & 14 & $47 \%$ \\
\hline Nexplanon implant & 0 & 0 \\
\hline Placement & & \\
\hline Intrapartum & 12 & $40 \%$ \\
\hline Postpartum & 18 & $60 \%$ \\
\hline Satisfaction with counseling & & \\
\hline Yes & 26 & $87 \%$ \\
\hline No & 4 & $13 \%$ \\
\hline Likelihood to recommend LARC & & $77 \%$ \\
\hline Very likely & 23 & $23 \%$ \\
\hline Somewhat likely & 7 & 0 \\
\hline Not likely & 0 & \\
\hline Would recommend against & 0 & \\
\hline & & \\
\hline
\end{tabular}

reduced menses as factors in selecting the hormonal IUD. Among the 55 with etonogestrel implant, 21 (38\%) cited ease of insertion and $10(18 \%)$ cited accessibility to arm implant. The majority of copper IUD users $(15,60 \%)$ desired to avoid hormonal treatment. Thirty women completed the acceptability survey (Table 1). Twenty-two (73\%) women confirmed that they were very likely to opt for an IUD again.

This global strategy of providing full-spectrum LARCs at no cost alongside tailored health education as the final step in the MCCP model was met with interest and initial satisfaction; it also helped to ensure that patients engaged with the local healthcare facilities, and to prevent pregnancy-related complications. Identification of further barriers to LARC uptake and longer-term follow-up to measure satisfaction with these methods will be useful in improving access to high-quality contraception.

\section{AUTHOR CONTRIBUTIONS}

ZA developed the LARC acceptability survey, analyzed the data and drafted the manuscript for submission. TS developed the model of the maternal continuum of care program, including study design, funding, enrollment and implementation. Both authors discussed the results and contributed to the final version of the manuscript.

\section{CONFLICTS OF INTEREST}

The authors have no conflicts of interest. 


\section{REFERENCES}

1. Kenya National Bureau of Statistics, Ministry of Health/Kenya, National AIDS Control Council/Kenya, Kenya Medical Research Institute, and National Council for Population and Development/Kenya. Kenya Demographic and Health Survey 2014. 2015. KNBS and ICF International. http://dhsprogram. com/pubs/pdf/FR308/FR308.pdf. Accessed October 30, 2019.
2. Butto $D$, Mburu S. Factors associated with male involvement in family planning in West Pokot County, Kenya. Univ J Public Health. 2015;34:160-168.

3. van Zijl S, van der Spuy ZM, Morroni C. A survey to assess knowledge and acceptability of the intrauterine device in the Family Planning Services in Cape Town, South Africa. BMJ Sex Reprod Health. 2010;36:73-78.

\title{
Uterine devascularization associated with compressive uterine suture (UD-CUS) in surgical treatment of postpartum hemorrhage
}

\author{
Álvaro Luiz Lage Alves ${ }^{1,2,3}$ | Janaína Campos Senra ${ }^{1, *}$ | Carolina Nogueira de São José ${ }^{3}$ | \\ Bruna Roque Ribeiro $^{3}$ | Rafaela de Souza Furtado ${ }^{1}$ | Lucas Barbosa Silva $^{1,2}$ | \\ Agnaldo Lopes Silva-Filho ${ }^{1}$
}

\footnotetext{
${ }^{1}$ Department of Obstetrics and Gynecology, Clinics Hospital, Federal University of Minas Gerais, Belo Horizonte, Brazil

${ }^{2}$ Department of Obstetrics and Gynecology, Sofia Feldman Hospital, Belo Horizonte, Brazil

${ }^{3}$ Department of Obstetrics and Gynecology, Faculty of Medical Sciences of Minas Gerais, Belo Horizonte, Brazil

${ }^{*}$ Correspondence

Janaina Campos Senra, Secretaria da Maternidade, Av. Prof. Alfredo Balena, 110, 4 Andar - Santa Efigênia, Belo Horizonte - MG, 30130-100, Brazil.

Email: janasenra@hotmail.com
}

KEYWORDS: Analytical; Diagnostic and therapeutic techniques; Fertility preservation; Healthcare; Near miss; Obstetric labor complications; Pregnancy complications; Puerperal disorders; Uterine atony; Uterine hemorrhage

Postpartum hemorrhage (PPH) is the most frequent severe birth complication and a significant cause of morbidity and preventable maternal deaths. ${ }^{1}$ The present study describes a new strategy for the preservation of the uterus, with a series of cases of PPH. Uterine devascularization associated with compressive uterine suture (UD-CUS) consists of vascular ligature in the ascendant branches of the uterine arteries (one in the right and one in the left side of the organ), as recommended by O'Leary, and two vertical braces of compressive uterine suture, as recommended by Hayman. ${ }^{2,3}$ In the Hayman technique, two separated compressive sutures are made: one in the right and one in the left half of the uterus exteriorized from the abdominal cavity. The needle passes through the uterus only once (from front to back or vice versa) in a sagittal plane for each suture at the inner top level of the cervix. Following on from this, each strap is then tied to the bottom of the uterus. The Hayman technique is useful for controlling bleeding in the uterine body ( $\mathrm{S} 1$ genital vascular territory) and preventing uterine atony and placental accretism restricted to the uterus (Fig. 1).
Between January 2014 and May 2017, the UD-CUS technique was performed on 41 patients who presented with $\mathrm{PPH}$ but did not recover with pharmacological treatment. The Sofia Feldman Hospital Research Ethics Committee approved the present study, and all patients signed a general informed consent form following hospital admission. At this institution, professionals have constantly been trained to perform the UD-CUS technique since it was developed in 2010.

Four cases had a multiple pregnancy (9.8\%) and 31 (75.6\%) had some type of comorbidity, with hypertensive disorders being the most frequent (34.1\%). The main PPH etiology was atony (82.9\%). There was accretism in three patients and uterine rupture in two. Four patients presented placental abruption and one of them developed Couvelaire uterus. Placenta previa was diagnosed in one case. Only one patient had PPH after vaginal delivery. Hemorrhage began during cesarean delivery in 33 cases (80.5\%) and after the procedure in seven cases (17.1\%).

All patients underwent pharmacological therapy and prophylactic antibiotic treatment before undergoing UD-CUS. The first line of therapy 


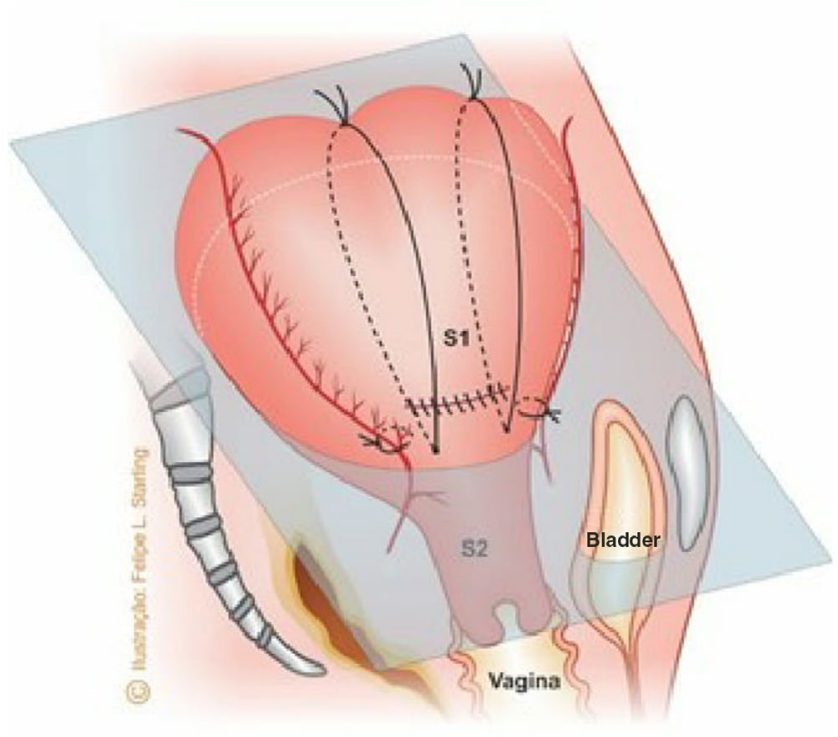

FIGURE 1 Schematic representation of uterine devascularization and compressive uterine suture (UD-CUS) technique according to the genital vascular territories.

included volume resuscitation, uterotonic agents (oxytocins, prostaglandins, and ergot alkaloids) and/or tranexamic acid. If the bleeding continued despite pharmacological therapy, the UD-CUS technique was considered.

The shock index (>0.9) revealed that 17 patients (41.5\%) suffered hypovolemic shock at some point of the procedure. Six patients were treated with vasoactive amines and 18 patients received blood transfusions. Blood loss was estimated by weighing surgical pads in only 12 patients (29.3\%) and varied between 480 and $3200 \mathrm{~mL}$ (mean of $1685.7 \pm 914.9 \mathrm{~mL})$.

The UD-CUS technique provided hemorrhagic control in 38 patients with a success rate of $92.7 \%$. In the cases of three patients in whom hemostatic control was not achieved, diagnosis and abdominal hysterectomy was required within hours or up to 3 days after the procedure. All patients survived, and six (14.6\%) had surgical complications (three cases of abdominal wall hematoma, two of pelvic hematoma and one case of puerperal endometritis).

Fotopoulou et al. recommend caution in combining the CUS technique with vascular ligatures due to the risk of uterine ischemic necrosis. ${ }^{4}$ When performing the UD-CUS technique, the compressive vertical braces are tied from the uterus fundus to the lower segment, thus preventing the uterine walls from collapsing and preventing interference in blood drainage. In this series, none of the patients developed an ischemic phenomenon in the short-term.

The success rate achieved using the UD-CUS technique in this study was comparable to previous studies. Chai et al. observed an increase in success rates of CUS from $66 \%$ to $74 \%$ when the procedure was performed in combination with devascularization techniques. ${ }^{5}$ Similarly, Kaya et al. described an improvement from $75 \%$ to $94.4 \%$ when devascularization techniques were combined with B-Lynch suture. ${ }^{6}$

The UD-CUS technique controlled bleeding in all three cases of accretism. This achievement could be attributed to mild accretism and blood irrigation from the S1 genital vascular territory. The UD-CUS technique is preferable in hemorrhages coming from this territory because vascular ligature is performed in the ascending branches of the uterine arteries.

Compared to the WHO Guideline recommendations, this technique is suitable for treatment of PPH, surgical maneuvers and other procedures (strong recommendation, very low-quality evidence). ${ }^{7}$

The present study does have some limitations, such as the lack of a control group and the fact that the sample was obtained by convenience. The strengths of the present study include the fact that the UD-CUS technique only requires four needle passages through the uterus, which facilitates execution, and that the success rate is comparable to that reported in previous literature.

In conclusion, this strategy integrates a set of surgical techniques that preserve the uterus, mainly in hemorrhages during cesarean delivery and in bleeding associated with S1 genital vascular territory.

\section{AUTHOR CONTRIBUTIONS}

All authors participated in the study's conception and design, in collecting and synthesizing data, and in drafting or revising the manuscript.

\section{ACKNOWLEDGMENTS}

We thank the Sofia Feldman Hospital, where this study was developed.

\section{CONFLICTS OF INTEREST}

The authors have no conflicts of interest.

\section{REFERENCES}

1. Main EK, Goffman D, Scavone BM, et al. National Partnership for Maternal Safety: Consensus bundle on obstetric hemorrhage. Anesth Analg. 2015;121:142-148.

2. O'Leary JL, O'Leary JA. Uterine artery ligation in the control of intractable postpartum hemorrhage. Am J Obstet Gynecol. 1966;94: 920-924.

3. Hayman RG, Arulkumaran S, Steer PJ. Uterine compression sutures: Surgical management of postpartum hemorrhage. Obstet Gynecol. 2002;99:502-506.

4. Fotopoulou C, Dudenhausen JW. Uterine compression sutures for preserving fertility in severe postpartum haemorrhage: An overview 13 years after the first description. J Obstet Gynaecol. 2010;30: 339-349.

5. Chai VY, To WW. Uterine compression sutures for management of severe postpartum haemorrhage: Five-year audit. Hong Kong Med J. 2014;20:113-120.

6. Kaya B, Tuten A, Daglar K, et al. B-Lynch uterine compression sutures in the conservative surgical management of uterine atony. Arch Gynecol Obstet. 2015;291:1005-1014.

7. WHO Guidelines Approved by the Guidelines Review Committee. WHO Recommendations for the Prevention and Treatment of Postpartum Haemorrhage. Geneva: World Health Organization; 2012. https://apps. who.int/iris/bitstream/handle/10665/75411/9789241548502_eng. pdf?sequence=1. Accessed December, 6, 2019. 